\title{
Clot lysis by gastric juice: an in vitro study
}

\author{
S E PATCHETT, HELEN ENRIGHT, N AFDHAL, W O'CONNELL, \\ AND D P O'DONOGHUE \\ From the Dept of Gastroenterology and Liver Unit and Dept of Haematology, St Vincent's Hospital, Dublin, \\ Ireland
}

SUMmary Gastric juice from patients with peptic ulcer disease and from patients with no upper gastrointestinal abnormality was studied in order to assess its effect on a formed fibrin clot. In both groups of patients gastric juice caused a marked increase in fibrinolysis as evidenced by a shortening of the euglobulin clot lysis time. This plasmin mediated fibrinolytic activity was found to be heat labile and only present in an acid environment. Addition of tranexamic acid or sucralfate to gastric juice almost completely reversed this effect, whereas pepstatin was only partially effective. It is probable that acid dependant proteases other than pepsin are responsible for the marked fibrinolysis. The ulcer healing agent sucralfate might be useful in those patients at risk of bleeding or rebleeding from active peptic ulcer disease.

Haemorrhage from active peptic ulcer disease remains a common and difficult therapeutic problem. Despite many new approaches in management, the mortality has changed little over the past 50 years. Approximately $10 \%$ of patients with acute upper gastrointestinal bleeding will die ${ }^{12}$ and the mortality rate may rise to over $30 \%$ in that subgroup requiring emergency surgery. ${ }^{3+}$ The poor haemostatic response seen in the bleeding upper gastrointestinal tract has been variously ascribed to increased gastrointestinal motility during haemorrhage, ${ }^{5}$ the marked vascularity and absence of autoregulation of local blood flow, and the adverse effects of both acid and pepsin on the coagulation cascade.' Moreover, dissolution of a formed fibrin clot is thought to be responsible for the very high incidence of rebleeding observed. Several investigators have identified fibrinolytic activity in gastric venous blood of patients with gastric haemorrhage ${ }^{\mathrm{x}}$ and in gastric juice of patients with erosive gastroduodenitis." ${ }^{11}$ Recent work has suggested a beneficial effect of specific antifibrinolytic agents in preventing rebleeding in acute upper gastrointestinal haemorrhage. ${ }^{11-1.3}$ In the present study we have assessed the degree of fibri-

Address for correspondence: D P O’Donoghue, Dept of Gastroenterology and Liver Unit, St Vincent's Hospital, Elm Park. Dublin 4. Ireland.

Accepted for publication 5 April 1989. nolytic activity in gastric juice in normal subjects and patients with active peptic ulcer disease and identified methods of manipulating this fibrinolysis.

\section{Methods}

COLLECTION OF GASTRIC JUICE

Studies were performed on samples of gastric juice obtained from patients referred for upper gastrointestinal endoscopy for evaluation of dyspeptic symptoms. Juice was obtained by aspiration through an air dried endoscope (Olympus Q10). It was immediately centrifuged at $500 \mathrm{rpm}$ for five minutes to remove particulate matter, the $\mathrm{pH}$ was measured, and the juice was subsequently stored at $-4^{\circ} \mathrm{C}$ pending further assessment.

Gastric juice obtained was divided into two groups depending on endoscopic findings: (A) Patients with no endoscopic abnormality (normal juice); (B) Patients with endoscopically proven active duodenal ulcers but no other endoscopic abnormality.

Gastric juice was not used if: (a) patients had known biliary or pancreatic disease; (b) patients were being treated with $\mathrm{H}_{2}$ blockers, anticholinergics, buffering agents, other ulcer healing drugs, or nonsteroidals; (c) gastric juice was obviously bile stained; (d) the $\mathrm{pH}$ of the gastric juice was greater than $2 \cdot 0$. 
ALTERATION OF GASTRIC JUICE:

At appropriate times, $50-100 \mu \mathrm{l} 0.1 \mathrm{~m}$ sodium hydroxide $(\mathrm{NaOH})$ was added to alter $\mathrm{pH}$ of gastric juice. Approximately $50-200 \mu \mathrm{l} \mathrm{NaOH}$ was required to raise the $\mathrm{pH}$ of $2 \mathrm{ml}$ aliquots of gastric juice to $4 \cdot 0$. Irreversible denaturation of gastric proteases was achieved by heating the juice to $80^{\circ} \mathrm{C}$ for 30 minutes, a process which does not alter the $\mathrm{pH}$ of gastric juice. On each occasion $\mathrm{pH}$ was measured before and after manipulation of gastric juice using the Bibby stick meter SMP1. (Bibby Science Products, Staffordshire, England.)

In addition to alkalinisation and heat denaturation, the effects of three specific pharmacological agents on the fibrinolytic potential of gastric juice were also assessed. The choice of agents was based on their known effects on the components of gastric juice or the fibrinolytic system. (1) Tranexamic acid: a specific plasminogen inhibitor was added at a concentration of $20 \mathrm{mg} / \mathrm{ml}$ of gastric juice. (2) Pepstatin: a specific pepsin inhibitor was used at a dose of $250 \mu \mathrm{l} / \mathrm{ml}, 10$ times that shown previously to inhibit peptic activity in gastric juice. ${ }^{1+}(3)$ Sucralfate: an aluminium sucrose sulphate complex, which has proven efficacy in peptic ulcer disease was added in a concentration of $12 \mathrm{mg} / \mathrm{ml}$ of gastric juice. This concentration was found from pilot studies to be the maximum which did not alter the $\mathrm{pH}$ of the gastric juice (mean change in $\mathrm{pH}-0 \cdot 06$, range $-0 \cdot(01-() \cdot(09)$.

ASSESSMENT OF FIBRINOIYTIC ACTIVITY

Plasmin mediated fibrinolytic activity was assessed using the euglobulin clot lysis time as described below.

EUGLOBULIN CLOT LYSIS TIME (ECLT)

Plasma was collected from 20 normal volunteers, pooled, anticoagulated with $0 \cdot 105$ mol sodium citrate, stored at $-70^{\circ} \mathrm{C}$ and thawed to $4^{\circ} \mathrm{C}$ immediately before use. Plasma fibrinolysis was assessed using the euglobulin clot lysis time a measure of plasminogen activator activity. The euglobulin clot lysis times were performed at $-4^{\circ} \mathrm{C}$ using the method of Walker.' ${ }^{\text {S }}$ Separation of the euglobulin fraction, containing fibrinogen, plasminogen, and plasminogen activator was achieved by dilution of $0.5 \mathrm{ml}$ chilled anticoagulated plasma in $9 \mathrm{ml}$ distilled water and acidification with $0.1 \mathrm{ml} 1 \%$ acetic acid. After standing at $4^{\circ} \mathrm{C}$ and centrifugation at $3000 \mathrm{rpm}$ for 10 minutes, the euglobulin fraction was precipitated, the supernatant discarded and the precipitate resuspended in borate buffer ( $\mathrm{pH} 9) .0 .2 \mathrm{ml}$ aliquots of the suspension were clotted with $0.2 \mathrm{ml}(0 .(2) 25 \mathrm{~m}$ calcium chloride and the time to clot lysis assessed visually at $37^{\circ} \mathrm{C}$. Tests were performed in duplicate and the means of the two values taken as the clot lysis time. Results were expressed as the time to clot lysis in minutes. Euglobulin clot lysis times were performed on $0.45 \mathrm{ml}$ plasma to which $0.05 \mathrm{ml}$ gastric juice had been added. The addition of gastric juice to plasma was found not to alter the final $\mathrm{pH}$ at which the euglobulin clot lysis times were performed (mean change in $\mathrm{pH}:(0 \cdot 14$, range: $0 \cdot(07-() \cdot 24)$. As a volume control, $0.05 \mathrm{ml}$ Tris buffer $(\mathrm{pH} \mathrm{7.2)}$ was added to plasma.

STATISTICAL ANALYSIS

Wilcoxon`s rank test for unpaired data (MannWhitney test) was used to analyse the euglobulin clot lysis time results obtained.

\section{Results}

UNALTERFD GASTRIC JUICE - EFFECT ON

PLASMA FIBRINOLYSIS

The mean euglobulin clot lysis time of pooled plasma was not significantly altered by the addition of $50 \mu \mathrm{l}$ of the volume control, Tris buffer $(p=0.48)$ (Table). However addition of $50 \mu \mathrm{l}$ gastric juice from normal patients very significantly shortened the euglobulin clot lysis time causing a $40.7 \%$ increase in fibrinolysis $(\mathrm{p}<0.0002)$ (Fig. 1). There was a similar reduction in the euglobulin clot lysis time when gastric juice from patients with proven peptic ulcer disease was added to pooled plasma with a $37.4 \%$ increase in fibrinolytic activity $(\mathrm{p}<0 \cdot 0002)$. There was no difference seen between gastric juice of normal patients and patients with peptic ulcer disease $(\mathrm{p}=0.48)$.

\section{ALTERED GASTRIC JUICE - EFFECT ON PLASMA FIBRINOLYSIS}

A rise of the $\mathrm{pH}$ of gastric juice to 4 almost completely abolished the fibrinolytic activity in gastric juice (mean euglobulin clot lysis time 217-1). This represents no significant increase in fibrinolysis $(p=0.45$, Fig. 2). Similarly when gastric juice which had been heated as described was added to plasma,

Table Mean and range of all euglobulin clot lysis times in minutes

\begin{tabular}{lrcl}
\hline & $n$ & Range (mins) & Mean \\
\hline Pooled plasma & & $204-273$ & $238 \cdot 3$ \\
TRIS buffer & 30 & $210-274$ & $235 \cdot 3$ \\
Gastric juice: A & 15 & $68-200$ & $140 \cdot 7$ \\
Gastric juice: B & 15 & $68-185$ & $149 \cdot 1$ \\
Gastric juice pH4 & 30 & $167-261$ & 217 \\
Heated juice & 30 & $180-26.5$ & $228 \cdot 2$ \\
()-1 m HCI & 4 & $204-222$ & 211 \\
Gastric juice + sucralfate & 14 & $176-250$ & $206 \cdot 1$ \\
Gastric juice + tranexamic acid & 8 & $178-245$ & 212 \\
Gastric juice+pepstatin & 11 & $103-210$ & $161 \cdot 2$ \\
\hline
\end{tabular}




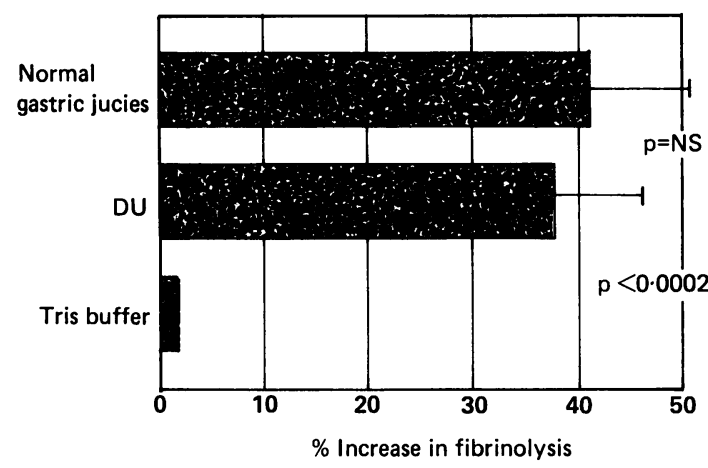

Fig. 1 Gastric juice from normal and peptic ulcer patients. Effect on plasma fibrinolysis (mean (SE)).

the euglobulin clot lysis time was not altered from control values. When acid alone was added to plasma either in the form of acidified Tris buffer, or $0.1 \mathrm{~m}$ $\mathrm{HCl}$, no change in euglobulin clot lysis time was detected.

ADDITION OF PHARMACOLOGICAL AGENTS TO GASTRIC JUICE - EFFECT ON PLASMA FIBRINOLYSIS

The results obtained when three pharmacological agents were added independently to gastric juice are displayed in the Table and Figure 3. Tranexamic acid completely reversed the fibrinolysis observed with normal gastric juice. Pepstatin, however, partially reversed fibrinolysis, and although the reduction was significant $(p<0.01)$ it was quantitatively considerably less than that observed with tranexamic acid or indeed heating or alkalinisation of gastric juice $(\mathrm{p}<0 \cdot 01)$.

Sucralfate caused a very marked inhibition of fibrinolysis. The $7 \cdot 5 \%$ increase in fibrinolysis caused

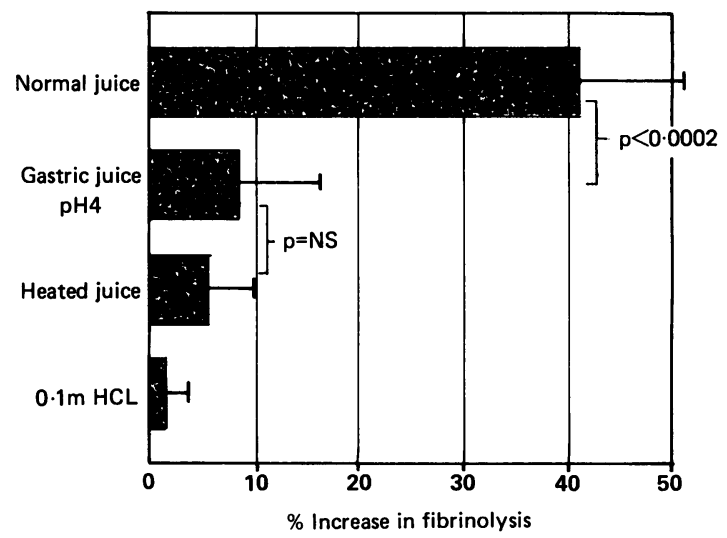

Fig. 2 Heated and alkalinised gastric juice. Effect on plasma fibrinolysis (mean (SE)).

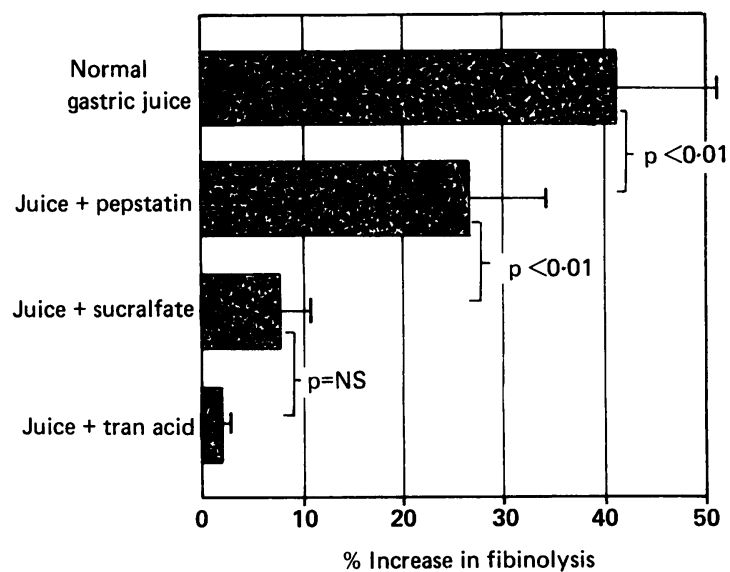

Fig. 3 Pharmacological agents added to gastric juice. Effect on plasma fibrinolysis (mean (SE)).

by gastric juice with Sucralfate was not significantly different from that observed with alkalinised or heated gastric juice $(p=0 \cdot 45)$.

\section{Discussion}

Rebleeding with 72 hours of upper gastrointestinal haemorrhage is a major clinical problem. As a result, great efforts have been made to develop treatment modalities which would successfully reduce the rebleeding rate and, as a result, the overall mortality.

Since Cox and coworkers identificd fibrinolytic activity in gastric veins of patients with upper gastrointestinal haemorrhage, ${ }^{, "}$ several workers have explored the role of fibrinolysis in bleeding peptic ulcer disease. Thompson discovered a plasmin-like enzyme, identified histochemically as plasmin in the gastric veins of patients with gastrointestinal haemorrhage. ${ }^{16}$ Nilsoon confirmed the presence of fibrinolytic activity in gastric juice of patients with erosive gastroduodenitis, but not in juice from normal patients."' These findings are in contrast, however, with those of O'Brien who found that fibrinolytic activity was a property of normal and haemorrhagic gastric juice. ${ }^{17}$

The finding, in this study, of very significant fibrinolytic activity in the gastric juice of normal patients and patients with non-bleeding peptic ulcer disease supports the suggestion of O'Brien that fibrinolytic activity of gastric juice is not confined to patients with upper gastrointestinal bleeding. ${ }^{17}$

The complete abolition of fibrinolysis by tranexamic acid confirms that this activity is plasmin mediated in contrast with what has been described as non-specific proteolytic activity. This activity could almost completely be abolished by raising the $\mathrm{pH}$ of 
the gastric juice to above 4 or alternatively by heat inactivation of gastric juice. This along with the finding that acid per se could not induce fibrinolysis suggests that the important activator of the fibrinolytic system is heat labile and active only at an acid $\mathrm{pH}$. Thus acidic gastric juice not only impairs clot formation, but also results in significantly greater clot lysis. Clearly, maintaining high intragastric $\mathrm{pH}$ in upper gastrointestinal bleeding is therefore theoretically justified. The failure of antacids and $\mathrm{H}_{2}$ blockers to clearly confer benefit in upper gastrointestinal bleeding may possibly be because of their inability to maintain a constant neutral environment particularly when the stomach contains food or blood. ${ }^{18}$ Although peptic activity is closely $\mathrm{pH}$ dependant and, in addition, has been implicated as a possible factor in causing clot lysis, the finding of an only partial though significant reduction of fibrinolysis by Pepstatin suggests that pepsin is at most playing only a minor role in plasminogen mediated clot lysis.

Sucralfate, an agent with proven efficacy in healing peptic ulcers, was also capable of profoundly inhibiting fibrinolysis in concentrations which did not significantly raise the $\mathrm{pH}$ of gastric juice. The mechanism by which sucralfate achieves this is not clear. It seems likely that a protease or range of proteases in gastric juice are capable of inducing clot lysis both by a specific plasmin mediated mechanism and by non-specific proteolytic digestion. Sucralfate has moderate buffering capacity and also is an efficient pepsin binder but its efficacy in inhibiting fibrinolysis is clearly not primarily a result of these properties. Nagashima and Terao have shown that sucralfate is capable not only of binding pepsin but also of binding a range of other proteins including fibrinogen and albumin. ${ }^{192}$ Sucralfate may therefore be able to inhibit this process by binding to, and inactivating one or more proteases in the proteolytic pathway. This aspect requires further investigation.

This study supplies strong theoretical support for the use of antifibrinolytic agents in bleeding peptic ulcer disease. Sucralfate would appear to have an advantage over Tranexamic acid in that it can also induce ulcer healing. In addition, the ability of Sucralfate to bind Pepsin suggests that it may also have a role in preventing non specific fibrinolysis. However, the effect of antifibrinolytic agents on clot formation as distinct from maintaining clot stability also requires further evaluation before these agents can be recommended without equivocation.

\section{References}

1 Allan R, Dykes P. A study of the factors influencing mortality rates from gastrointestinal hacmorrhage. QJ Med 1976; 45: 533-50.

2 Crook J, Gray L Jr. Nance F, Cohn J. Upper gastrointestinal blecding. Ann Surg 1972; 175: 771-9.

3 Hunt PS. Francis JK. Hanskey J, et al. Reduction in mortality from upper gastrointestinal hacmorrhage. Med J Aust 1983; ii: 552-5.

4 Vellacott K. Dronficld M. Atkinson M. Langman M. Comparison of surgical and medical management of blecding peptic ulcers. Br Med J 1982; 284: 548-50.

5 Bodi T, Wirts CW. Tocantins LM. Local environmental factors affecting haemostasis in blecding from the upper gastrointestinal tract. Prog Haematol 1956; 1: 221-48.

6 Davenport HW. Physiology of the digestive tract. Chicago: Year-book Medical Publishers Inc, 1968: 129.

7 Green F Jr, Kaplan M, Curtis L, Levine P. Effect of acid and pepsin on blood coagulation and platelet aggregation. A possible contributor to prolonged gastroduodenal mucosal hacmorrhage. Gastroenterology 1978; 74: $38-43$.

$8 \operatorname{Cox}$ H. Poller L. Thompson J. Gastric fibrinolysis. A possible aetiological link with peptic ulcer. Lancet 1967; i: $1300-2$.

9 Cox H, Poller L, Thompson J. Evidence for the release of gastric fibrinolytic activity into peripheral blood. Gut 1969; 10: 40-7.

10 Nilsson IM, Bergentz SE, Hender U, et al. Gastric fibrinolysis. Thromb Haemost 1975; 34: 409-17.

11 Stael von Holstein CCS, Eriksson SBS, Kallen R. Tranexamic acid as an aid to reducing blood transfusion requirements in gastric and duodenal blecding. $\mathrm{Br} \mathrm{Med} \mathrm{J}$ 1987; 297: 7-10.

12 Biggs J, Hugh T, Dodds A. Tranexamic acid and upper gastrointestinal hacmorrhage - a double blind trial. Gut 1976; 17: 729-34.

13 Barer D, Ogilvic A, Henry O, et al. Cimetidine and Tranexamic acid in the treatment of acute upper gastrointestinal tract blecding. $N$ Engl J Med 1983; 308: 1571-5.

14 Berstad A, Holm HA, Kittang E. Experience with antipcptic agents. Scand J Gastroenterol 1979; 14 [suppl 55]: $121-3$.

15 Walker ID, Davidson JF. Blood coagulation and haemostasis - a practical guide. 3rd ed. Edinburgh: Churchill Livingstone, 1985: 229.

16 Thompson J. Fibrinolysis and the stomach. Biochemical identification of the 'plasmin like' enzyme. Thromb Diath Haemorrh 1974; 31: 291-8.

17 O'Brien T. Hadley H. Irving M. Fibrinolytic activity in gastric venous blood. Gastroenterology 1979; 76: 50914.

18 Merki HS. Witzel L, Kaufman D, et al. Continuous intravenous infusions of famotidine maintain high intragastric pH in duodenal ulcer. Gut 1988; 29: 453-7.

19 Nagashima R, Yoshida N, Terao N. Sucralphate, a basic aluminium salt of sucrose sulphate. Arzneim Forsch [Drug Res/ 1980; 30: 73-6.

20 Terao N, Yoshida N, Nagashima R. Inhibition of peptic hydrolysis of fibrinogen by sucrose sulphate. Arzneim Forsch /Drug Res/ 1980; 30: 76-8. 